\section{revisão}

\author{
Antonela F.A. Siqueiva \\ Dulcinéia S.P. Abdalla \\ Sandra R.G. Ferreiva
}

\author{
Disciplina de Endocrinologia \\ (AFAS), Universidade Federal de \\ São Paulo - UNIFESP/EPM; \\ Faculdade de Ciências \\ Farmacêuticas (DSPA) e \\ Departamento de Nutrição da \\ Faculdade de Saúde Pública \\ (SRGF), Universidade de São \\ Paulo - USP, São Paulo, SP.
}

Recebido em 20/12/05

Aceito em 17/01/06

\title{
LDL: da Sindrome Metabólica à Instabilização da Placa Aterosclerótica
}

\section{RESUMO}

A dislipidemia da síndrome metabólica (SM) confere elevado risco cardiovascular e caracteriza-se por aumento dos triglicérides, diminuição da HDL e alterações qualitativas da LDL, tornando-a mais aterogênica, como a LDL pequena e densa. LDLs modificadas (LDLm) foram detectadas in vivo no plasma e em placas ateroscleróticas. Uma pequena porcentagem do total de LDLs plasmáticas apresenta maior carga negativa na superfície (LDL(-)), sendo uma sub-população heterogênea de partículas com maior poder de agressão ao endotélio. Origina-se da oxidação, glicação ou outros processos que alteram sua composição química, estando aumentada em indivíduos diabéticos, hipercolesterolêmicos e naqueles com doença isquêmica cardíaca. A LDLm, ao ser fagocitada pelo receptor scavenger do macrófago, transforma-o numa célula espumosa e inicia uma reação imune-inflamatória. A participação da LDLm no processo aterogênico continua até a ruptura da placa e trombogênese, quando ela induz apoptose em células endoteliais e musculares lisas, aumenta a produção de metaloproteinases que digerem a matriz, fragilizando a cápsula, e exacerba a inflamação que concorre para o desenvolvimento do trombo. O aprimoramento dos ensaios laboratoriais para a LDLm permitirá maior aplicabilidade clínica, melhorando o poder preditivo de eventos cardiovasculares em relação ao perfil lipídico convencional e demais fatores de risco presentes na SM. (Arq Bras Endocrinol Metab 2006;50/2:334-343)

Descritores: Lipoproteína de baixa densidade; Oxidação; Síndrome metabólica; Aterogênese

\section{ABSTRACT}

\section{LDL: From Metabolic Syndrome to Instability of the Atherosclerotic Plaque.}

The dyslipidemia of the metabolic syndrome (MS) confers an elevated cardiovascular risk and is characterized by increased concentrations of triglycerides, decreased $\mathrm{HDL}$-cholesterol and qualitative alterations in LDL which renders it more atherogenic, like the small dense LDL. Modified forms of $L D L(m L D L)$ have been detected in vivo in the plasma and atherosclerotic plaques. A minor fraction of the total LDL has an electronegative charge and is represented by a heterogenic subpopulation of particles $(\operatorname{LDL}(-))$, with higher potential to induce endothelial injury. It could be derived from oxidation, glication or other processes that alter its chemical composition and is increased in diabetic, hypercholesterolemic subjects, and in those with established coronary artery disease. $\mathrm{mLDL}$ are internalized by macrophages through scavenger receptors, originating foam cells and inducing an immune-inflammatory reaction. In the atherosclerotic process, the action of $\mathrm{mLDL}$ continues until plaque rupture and thrombogenesis, when it promotes apoptosis in endothelial and smooth muscle cells, and activates matrix metalloproteinases, weaken the fibrous cap, and further enhance the inflammatory process that ends 
in the thrombus formation. Development of new laboratory methods is necessary to enhance the clinical applicability of $\mathrm{mLDL}$ and the predictive power of the conventional lipid profile and other cardiovascular risk factors of the MS. (Arq Bras Endocrinol Metab 2006;50/2:334-343)

Keywords: Low density lipoprotein; Oxidation; Metabolic syndrome; Atherogenesis

É NOTÁVEL O ESPAÇO QUE A SÍNDROME metabólica (SM) tem ocupado na literatura nas últimas décadas. Isto ocorre por conferir aos seus portadores um risco cardiovascular dos mais elevados e pelo fato de reunir uma série de fatores ou condições de risco em ascensão nas populações. $\mathrm{O}$ aumento da expectativa de vida contribui para que tais fatores se tornem mais freqüentes e, dessa forma, justifica considerar a SM como uma grande preocupação em termos de saúde pública. Entre esses fatores, agrupados sob o denominador comum da resistência à insulina (RI), estão a obesidade visceral, a intolerância à glicose, a hipertensão arterial sistêmica, a hipertrigliceridemia e os níveis baixos de HDL (lipoproteína de alta densidade), sendo estes os parâmetros diagnósticos da síndrome propostos pelo National Cholesterol Education Program - Adult Pannel III - NCEPATPIII (1). Outros especialistas e sociedades científicas $(2,3)$ também propuseram critérios diagnósticos, não existindo, ainda, um conjunto deles que seja universalmente aceito. Recentemente, a International Diabetes Federation - IDF divulgou uma proposta que seria uma "definição de consenso" (4), que valoriza a presença de obesidade central dentre os critérios, diferenciando os valores de circunferência da cintura segundo a etnia (caucasóide, asiática não-japonesa e japonesa).

Apesar da fração LDL (lipoproteína de baixa densidade) do colesterol circulante não constar dos critérios, analisando mais profundamente as alterações lipídicas nos estados de RI observa-se aumento no número de partículas pequenas e densas desta lipoproteína (LDLpd), reconhecidamente mais aterogênicas do que as partículas maiores (5). Considerando que a mortalidade destes indivíduos decorre dos eventos aterotrombóticos, este estudo se propôs a revisar o papel da LDL na patogênese da aterosclerose, assim como a resgatar a participação desta partícula, desde as primeiras modificações qualitativas que surgem na vigência da $S M$, até o momento da instabilização da placa e trombo-embolismo. Sendo amplo o espectro de manifestações da $\mathrm{SM}$, vários mecanismos, dentre eles a glicação e o estresse oxidativo, atuam em inten- sidades diversas para modificar as LDLs, comprometendo sua remoção (clearance) da circulação e aumentando seu potencial de agressão ao endotélio. $\mathrm{O}$ entendimento das modificações nas lipoproteínas circulantes é muito relevante para o planejamento terapêutico destes indivíduos, podendo contribuir, em conjunto com outras medidas, para a mudança no quadro de morbi-mortalidade das populações de alto risco para a síndrome.

\section{Alteraçōes lipídicas da Síndrome Metabólica}

Para a compreensão do fenótipo lipídico aterogênico presente na SM, é preciso inicialmente salientar a importância da adiposidade visceral. $\mathrm{O}$ tipo de adipócito que se encontra neste sítio tem intensa atividade lipolítica, liberando grandes quantidades de ácidos graxos livres (AGLs) na circulação portal e sistêmica. O fluxo aumentado de AGLs no fígado resulta em diminuição da captação hepática de insulina, inibindo sua ligação ao receptor e sua degradação, causando hiperinsulinemia sistêmica. Também pelo excesso de AGLs, há redução na degradação da apolipoproteína B100 (ApoB100), causando maior secreção hepática de VLDLs (lipoproteínas de densidade muito baixa) (6).

A proteína de transferência de ésteres de colesterol (CETP) contribui para a remoção do colesterol dos tecidos periféricos de volta ao fígado, e uma condição pró-aterogênica se estabelece quando sua ação está aumentada, como na RI: triglicérides (TGs) são transferidos das VLDLs para as LDLs e HDLs em troca de ésteres de colesterol. Então, a lipase hepática, cuja atividade também está aumentada, hidrolisa as LDLs e HDLs, gerando LDLpd, além de resultar em hipertrigliceridemia e diminuição da HDL2, a subpopulação de HDLs com maior atividade anti-aterogênica no plasma $(7,8)$.

Resumindo, o perfil lipídico da SM é caracterizado por hipertrigliceridemia, diminuição das HDLs e produção de LDLpd. Para fins diagnósticos, apenas as concentrações séricas elevadas de TGs e baixas de HDL fazem parte dos parâmetros sugeridos pelo NCEP (1). No entanto, a LDLpd vem sendo apontada como importante fator de risco para a doença isquêmica cardíaca (9). O Quebec Cardiovascular Study, usando eletroforese em gel de agarose para separar as LDLs de acordo com o tamanho da partícula, verificou que indivíduos portadores de LDL com diâmetro < 256,4 A e concentrações plasmáticas de ApoB superior a $120 \mathrm{mg} / \mathrm{dl}$ apresentavam risco cardiovascular marcadamente elevado (10). Na mesma linha, os resultados do Familial Atherosclerosis Treatment Study mostraram que o preditor mais potente da regressão da estenose 
coronariana, induzida pelo tratamento agressivo dos lipídios séricos, foi a diminuição da densidade das LDLs e não a mudança nos níveis séricos da partícula (11).

\section{A lipoproteína de baixa densidade}

As lipoproteínas plasmáticas são partículas constituídas por uma parte central de lipídios hidrofóbicos, circundados por uma monocamada de lipídios polares e apoproteínas. São sintetizadas e secretadas pelo fígado (VLDL) ou intestino (quilomícrons), ou resultam de transformações sofridas por estas partículas, como é o caso da IDL (lipoproteína de densidade intermediária), LDL e HDL. Têm duas funções principais: solubilizar os lipídios no plasma e regular o movimento destes em sua entrada e saída de células-alvo e tecidos específicos. Classificam-se de acordo com sua densidade, e a LDL encontra-se na faixa entre 1019 a 1063 $\mathrm{g} / \mathrm{ml}(12,13)$.

A hidrólise da VLDL, catalisada pela lipase lipoprotéica plasmática ou pela lipase hepática, remove TGs destas partículas, formando inicialmente a IDL e a seguir a LDL. Neste processo, ocorre simultaneamente a transferência de apolipoproteínas para a HDL, restando uma única molécula de ApoB100 na superfície da LDL. Esta lipoproteína é a principal transportadora do colesterol sérico (cerca de 70\% circula ligado a ela) e fornecedora deste lipídio para os tecidos extra-hepáticos, por meio da sua ligação ao receptor de LDL na membrana plasmática. Apresenta, em média, $22 \mathrm{~nm}$ de diâmetro, sendo composta de uma parte central com moléculas de colesterol esterificado (35\% da partícula) e de TGs (10\%), ao redor da qual há uma capa de colesterol livre, fosfolipídios e apoproteínas. Sua principal apoproteína é a ApoB100, mas também possui traços de Apo-E. A meia-vida plasmática é de cerca de dois a três dias, e seu clearance ocorre por meio da ligação das apolipoproteínas com o receptor de LDL, principalmente nos hepatócitos $(75 \%)$, mas também em outros tecidos extra-hepáticos $(12,13)$.

\section{A LDL na Síndrome Metabólica}

\section{Modificações na LDL nativa (LDLn)}

As modificações na LDLn vêm sendo estudadas por vários enfoques metodológicos, resultando em diversas classificações e numa nomenclatura complexa para expressar as diferentes alterações físico-químicas que a partícula pode sofrer. A oxidação é um dos processos de modificação mais estudados. Pode ser induzida in vitro, por metais de transição como ferro e cobre (14). A LDL assim oxidada apresentou características pró- aterogênicas, o que despertou grande interesse sobre sua existência in vivo. Identificou-se a presença de LDL oxidada (LDLox) em placas ateroscleróticas tanto em modelos animais como em humanos (15). No entanto, teoricamente não seria encontrada LDLox circulante, pois a LDLn estaria protegida pelos antioxidantes plasmáticos, como a vitamina $\mathrm{C}$.

$\mathrm{Na}$ década de 1980, numerosos investigadores estudaram a LDLox in vitro. Henriksen e cols. verificaram que, após a incubação da LDLn com células endoteliais por 12 a 18 horas, a partícula resultante era captada por macrófagos em cultura, 3 a 10 vezes mais rapidamente que a LDLn (16). Células musculares lisas exerciam efeito semelhante sobre a partícula (17) e os próprios monócitos e macrófagos também podiam induzir tais modificações (18). O processo era decorrente da ação de espécies reativas de oxigênio (ROS) e nitrogênio (RNS), liberadas das células, sobre os ácidos graxos poliinsaturados da partícula, desencadeando peroxidação lipídica. A ausência dos antioxidantes plasmáticos para se contrapor às ROS tornava $\mathrm{O}$ processo oxidativo da LDL in vitro mais intenso, nãofisiológico. A LDL assim gerada apresentava as seguintes características, quando comparada à LDLn:

- Aumento da carga negativa;

- Aumento da densidade;

- Conteúdo aumentado de lisolecitina, óxidos de colesterol e hidroperóxidos lipídicos e conteúdo diminuído de ácidos graxos poliinsaturados, como conseqüência da oxidação de seus lipídios;

- Fragmentação da ApoB100;

- Atividade quimiotática para monócitos circulantes e inibitória para a migração de macrófagos da parede arterial para a circulação;

- Citotoxicidade aumentada;

- Menor taxa de captação pelo receptor da LDLn; ávida captação pelos receptores scavenger dos macrófagos (19).

Em estudos com a LDLox in vivo, foi sugerido que o processo oxidativo ocorresse em microambientes da parede arterial (na ausência de antioxidantes plasmáticos) com posterior retorno da partícula modificada à circulação (19). Atualmente se aceita que uma pequena proporção de partículas seja modificada ainda na circulação e que a oxidação continue após a entrada da LDL na íntima das artérias, em ambiente pró-oxidante (20). Durante a modificação oxidativa, a parte protéica da LDL reage com produtos secundários da lipoperoxidação, como, por exemplo, o malondialdeído (MDA), formando MDA-LDL, cuja quantificação tem sido usada para avaliar o estresse oxidativo in vivo (21). 
A LDL, uma vez oxidada, tem aumento da carga negativa na sua superfície. Em 1988, Avogaro e cols. (22) isolaram, por cromatografia de troca aniônica, uma fração da LDL do plasma de indivíduos normolipidêmicos com maior carga negativa, denominando-a de LDL eletronegativa, $[\operatorname{LDL}(-)]$, a qual apresentava características semelhantes à LDLox. Mas, convém assinalar que o pool de $\operatorname{LDL}(-)$ não é formado exclusivamente por LDLox, mas também origina-se de outros processos, como a glicação nãoenzimática, o enriquecimento em ácidos graxos nãoesterificados (NEFAs), modificações enzimáticas por fosfolipases, reação cruzada com a hemoglobina e outros mecanismos ainda não identificados (23).

A subfração de LDL(-) constitui-se em um pool de partículas heterogêneas em tamanho e com tendência a agregação, com conteúdo diminuído de fosfolipídios e vitamina $\mathrm{E}$, e aumento de colesterol livre e proteínas, quando comparada à LDLn (22). Diferente da LDL oxidada in vitro, a LDL(-) não apresenta fragmentação da ApoB e outras alterações decorrentes de uma oxidação excessiva. Esta observação deu origem ao termo "LDL minimamente modificada" (LDLmm), empregado por alguns grupos ao se referirem à LDL de Avogaro (24).

Baseando-se em outras propriedades físicoquímicas da partícula, como tamanho e densidade, Austin e cols. (25) estabeleceram dois fenótipos principais relacionados às LDLs: padrão A - LDLs grandes e leves; e padrão B - LDLs pequenas e densas. Os estudos clínicos e epidemiológicos que abordam a relação entre presença de LDL modificada (LDLm) com SM e aterogênese têm comumente empregado esta classificação. A LDL(-) está contida na subfração de LDLpd, podendo ser responsável pela maior susceptibilidade à oxidação desta sub-população de partículas (26).

Em recente revisão, Sánchez-Quesada e cols. (23) ressaltaram o aumento da proporção da LDL(-) no plasma de indivíduos com elevado risco cardiovascular, como os portadores de diabetes mellitus (DM), hipercolesterolemia familiar ou hipertrigliceridemia, e também naqueles em hemodiálise ou com doença coronariana estabelecida. Em população diabética, observou-se maior proporção de LDL(-) nos portadores de DM tipo 1 descompensado, quando comparados a controles saudáveis. No entanto, a estratificação em fenótipos $\mathrm{A}$ ou $\mathrm{B}$ foi semelhante entre os grupos e a LDL dos indivíduos diabéticos mostrou-se resistente à oxidação. Após três meses de tratamento insulínico intensivo, a LDL(-) diminuiu de $19,0 \%$ para $13,8 \%(\mathrm{p}<0,05)$, porém não houve mudanças no padrão fenotípico ou na susceptibilidade à oxidação, sugerindo a glicação como geradora de LDL(-) nestes pacientes (27). Neste processo há conjugação não-enzimática da $\mathrm{ApoB}$ com a glicose, e a partícula formada não é reconhecida pelo receptor de LDL, mas sim por receptores scavenger (19). Portadores de DM tipo 2 também apresentaram maior proporção de LDL(-) em relação aos controles saudáveis $(19,0 \%$ versus $14,3 \%$; $<0,05)$; no entanto, estas LDLs eram rapidamente oxidadas in vitro. Após otimização do controle glicêmico, não se observaram mudanças na porcentagem de $\operatorname{LDL}(-)$, sugerindo que a oxidação, mais do que a glicação não-enzimática, está relacionada à origem de $\operatorname{LDL}(-)$ nestes indivíduos (28).

Dessa forma, conclui-se que as LDLs são um grupo heterogêneo de partículas que variam em tamanho, densidade, composição química e carga elétrica, e que várias classificações vêm sendo propostas na tentativa de elucidar seu papel na aterogênese. Não há estudos que enfoquem a relação da $\operatorname{LDL}(-) \operatorname{com}$ a SM, mas a presença de LDL fenótipo B no espectro de anormalidades da síndrome já foi estabelecida tanto em estudos caso-controle $(9,25)$ como prospectivos $(5,10)$, mostrando associação consistente entre LDLpd e doença isquêmica cardíaca. Porém, na prática clínica, determinação qualitativa da LDLm - oxidada, eletronegativa ou padrão B - não é habitualmente solicitada devido a limitações técnicas e indisponibilidade na rotina laboratorial. É provável que ela venha a acrescentar poder preditivo de eventos cardiovasculares no homem.

\section{Destino metabólico da LDLm - receptores scavenger}

Por se tratar de células fagocíticas, os macrófagos têm como função primordial a captação e neutralização de antígenos não-próprios ao organismo. A remoção da LDLm faz parte de sua função protetora precoce durante a resposta inflamatória, minimizando também os efeitos nocivos dos lipídios oxidados ao endotélio. Porém, em contraste com a maioria das células onde a expressão do receptor de LDL é regulada pelo conteúdo intracelular de ésteres de colesterol, a expressão dos receptores scavenger para as formas modificadas da partícula nos macrófagos não é inibida pela alta concentração de colesterol intracelular. A manutenção de altas concentrações de LDLm culmina com a formação das células espumosas, macrófagos carregados de lipídios tóxicos, que exercem ação inflamatória e induzem proliferação celular no espaço sub-endotelial (29). Como a LDLox é imunogênica, há produção de auto-anticorpos que formam 
imunocomplexos com a partícula modificada, os quais são internalizados pelo receptor $\mathrm{Fc}$ gama dos macrófagos, induzindo também a formação de células espumosas (30).

Diversas classes de moléculas vêm sendo identificadas nos macrófagos como receptores scavenger para a LDLm. Alguns tipos não são exclusivos dos macrófagos, como é o caso do receptor de LDLox semelhante à lectina 1 (LOX-1), recentemente descrito como uma glicoproteína de membrana encontrada também em células endoteliais e células musculares lisas $(31)$.

O estudo do LOX-1 no processo aterosclerótico vem sendo aprofundado, revelando achados interessantes. Mediadores de inflamação, endotoxinas bacterianas, angiotensina-II, força de cisalhamento de fluxo e a própria LDLox aumentam a expressão do LOX-1 no endotélio, sugerindo que ele possa estar expresso localmente nas placas ateromatosas em formação, onde há estímulos mecânicos e inflamatórios. Dentre os ligantes para o LOX-1 estão lipídios oxidados da LDL, e também células apoptóticas, confirmando sua função de scavenger (31). A captação de LDLox por meio do LOX-1 modula algumas funções celulares. Ao promover estresse oxidativo em células endoteliais, ativa o fator de transcrição nuclear NFKB, cuja função é promover a transcrição de genes pró-inflamatórios (32). Em cultura de células musculares lisas, a internalização da LDLox induz apopose celular, sendo possível que, in vivo, este processo possa fragilizar a capa fibrótica da placa (33). LOX-1 pode ser encontrado em forma solúvel no plasma. Seus níveis circulantes elevados podem refletir a atividade da doença aterosclerótica (31), mas ainda não há estudos avaliando o papel do LOX-1 como marcador de risco cardiovascular na SM.

\section{Resposta imune à LDLm: geração de auto- anticorpos}

Crescem as evidências experimentais de uma participação da imunidade adquirida no processo aterosclerótico. Auto-anticorpos direcionados à LDLox foram detectados no plasma de indivíduos com fatores de risco ou doença cardiovascular estabelecida, e imunocomplexos formados a partir destes com a LDLox foram encontrados em placas de ateroma. Porém, o papel deste sistema imune na origem e progressão da doença aterosclerótica foi pouco estudado e a importância dos anticorpos como marcadores de risco cardiovascular ainda não-estabelecida (34).

As modificações sofridas pela LDL, mesmo sendo sutis, transformam-na em uma partícula alta- mente imunogênica. Fosfolipídios contendo ácidos graxos poliinsaturados, após sofrerem modificações oxidativas, ligam-se a resíduos de lisina na $A p o B$, gerando neo-epitopos antigênicos, desencadeando, dessa forma, uma resposta auto-imune. Em modelos animais, títulos plasmáticos elevados de anti-LDLox correlacionaram-se fortemente com idade (35) e dieta rica em colesterol (36). Em humanos, anticorpos antiLDLox foram detectados no plasma tanto de indivíduos com elevado risco cardiovascular (37) como em populações aparentemente saudáveis (38). Salonen e cols. relataram que títulos basais de anti-MDA-LDL foram excelente preditor do aumento da espessura íntima-média da artéria carótida, em um período de dois anos, em população finlandesa de homens saudáveis (39). Os estudos relacionando os anticorpos anti-LDLox com doença cardiovascular, ou seus fatores de risco, têm encontrado resultados conflitantes (34). Cabe ressaltar que críticas à detecção da LDLox baseiam-se no fato de que a oxidação da partícula poderia ocorrer durante o processo analítico e, sendo assim, a determinação dos seus auto-anticorpos seria um indicador mais fiel do que ocorre in vivo (20).

Em indivíduos com DM tipo 1, Lopes-Virella e cols. (37) encontraram relação inversa entre a presença de anticorpos livres anti-LDLox no plasma e surgimento de doença arterial coronariana (DAC). O mesmo estudo mostrou correlação direta entre imunocomplexos formados por estes anticorpos com a LDLox e DAC. A formação destes imunocomplexos parece ser importante no processo aterogênico de indivíduos diabéticos, uma vez que sua captação por receptores Fc gama específicos dos macrófagos induz um massivo acúmulo de colesterol nestas células, e a liberação de citocinas inflamatórias (30).

Estudos ligando a resposta imune à LDLox e o risco cardiovascular conferido pela SM são muito escassos. Até o presente, apesar de vários relatos sobre anticorpos anti-LDLox presentes em indivíduos com DM (37) ou com DCV $(40,41)$, pouco ou nada se pode dizer a respeito da formação de auto-anticorpos como um dos mecanismos aterogênicos da SM.

\section{Reação inflamatória à LDLm: produção de citocinas}

A aterosclerose é considerada uma doença imuneinflamatória, na qual células imunocompetentes, tais como monócitos/macrófagos e linfócitos T, estão presentes em todos os estágios da lesão. A disfunção endotelial, considerada como passo inicial na aterogênese, caracteriza-se por uma resposta inflamatória da parede vascular à injúria, ou seja, um conjunto de 
reações celulares e moleculares a diversos agressores, dentre eles o aumento da concentração de LDL e a presença de LDLm (29). Esta lipoproteína atua no endotélio induzindo a expressão gênica do fator estimulador de colônia de macrófagos e da proteína quimiotática para monócitos 1 (MCP-1), estimulando a replicação de macrófagos no espaço sub-endotelial e recrutando mais células inflamatórias para a lesão em formação (29). Ao captarem LDLm, os macrófagos estimulam a proliferação de linfócitos $\mathrm{T}$ helper. Seguese uma conversa cruzada entre estas duas populações de células, cada qual produzindo citocinas específicas, implementando o processo inflamatório crônico característico da doença (34).

A produção de citocinas pelas células endoteliais sob estímulo da LDLm foi demonstrada em estudos in vitro. Sanchez-Quesada e cols. mostraram que a $\operatorname{LDL}(-)$ em baixas concentrações induziu a liberação de MCP-1 e interleucina 8 (IL-8) pelas células endoteliais $(42,43)$. Estas citocinas, por sua vez, atraem respectivamente monócitos e linfócitos, perpetuando o ciclo inflamatório. Os autores apontam para a possibilidade de que, in vivo, uma pequena quantidade de LDL(-) desencadeie resposta inflamatória no endotélio, e que este efeito seja mais pronunciado em indivíduos hipercolesterolêmicos, nos quais a proporção de $\operatorname{LDL}(-)$ no plasma chega a ser oito vezes maior que nos indivíduos normolipidêmicos (43).

A bioatividade da partícula de LDLm, que confere seu poder de agressão ao endotélio, depende, em parte, de seu conteúdo de lipídios oxidados. A quantidade de lipídios bioativos é regulada por enzimas, entre as quais destaca-se a acetil-hidrolase do fator ativador de plaquetas (PAF-AH) e a fosfolipase A2 (PLA2), associadas à lipoproteína. A atividade da PAF-AH é mais pronunciada na sub-população de LDL(-) (44) e também nas LDLpd (20), sugerindo um papel pró-aterogênico para a enzima. No entanto, ao exercer sua ação, a PAF-AH hidrolisa fosfolipídios oxidados, removendo o excesso de NEFAs oxidados da partícula, teoricamente diminuindo seu potencial inflamatório. Estudos in vivo com inibidores específicos desta fosfolipase serão necessários para certificar seu papel pró ou anti-inflamatório no processo aterogênico $(20,44)$.

É possível que a indução de citocinas pela LDL(-), decorrente da ação dos seus lipídios oxidados, ocorra através da cascata inflamatória dependente do NFкB. Fosfolipídios oxidados, como a lisolecitina e o ácido lisofosfatídico, induzem a expressão de genes pró-inflamatórios em células endoteliais, ativando o
NFкB (20); os NEFAs, presentes em grande quantidade na LDL(-), também são capazes de ativá-lo, atuando como mediadores da inflamação (20), pelo fato de os genes que codificam citocinas, como a MCP-1 e a IL8 , conterem sítios de ligação para este fator de transcrição (42). De fato, o NFkB em sua forma ativada foi detectado em áreas ateromatosas (45). Sabendo-se que a insulina apresenta propriedades anti-inflamatórias, atribuídas em parte à sua capacidade de inibir a ação do NFKB (46), pode-se supor que as vias inflamatórias ativadas por ele possam ser um elo entre a oxidação lipídica, inflamação e aterosclerose em estados de resistência à insulina.

\section{Citotoxicidade da LDLm}

A indução direta de apoptose nas células endoteliais é um dos mecanismos pelos quais a LDLox in vitro revela efeitos pró-aterogênicos. Para esclarecer se a LDL in vivo apresenta tais efeitos, Sevanian e cols. isolaram a fração $\operatorname{LDL}(-)$ do plasma de macacos e verificaram que ela exibe efeitos citotóxicos em cultura de células endoteliais, não observados com a LDLn (47). Outros pesquisadores também verificaram efeitos citotóxicos exacerbados da LDL(-) em cultura de células endoteliais de veia umbilical humana (48). Recentemente, buscando elucidar os mecanismos próapoptóticos da LDL circulante, Chen e cols. isolaram cinco frações da LDL do plasma de indivíduos hipercolesterolêmicos, de acordo com a carga elétrica da partícula, denominando de L5 a fração mais eletronegativa. A subfração L5, moderadamente oxidada, promoveu a apoptose de células vasculares através da supressão da transcrição do fator de crescimento de fibroblastos tipo 2 (FGF-2), peptídeo promotor da sobrevivência celular (49).

\section{Desenvolvimento e instabilização da placa aterosclerótica - papel da LDLm nos eventos cardiovasculares agudos}

Desde a disfunção endotelial até a ruptura de lesões na parede vascular, pode-se observar a participação ativa da LDLm como um agente pró-aterogênico. Inicialmente, os lipídios oxidados da partícula atuam como agressores ao endotélio e a resposta compensatória à injúria altera sua homeostase. A primeira lesão observada na parede arterial é a estria gordurosa, composta principalmente de macrófagos, transformados em células espumosas após a internalização da LDLox. Esta reação inflamatória busca inicialmente combater os agentes agressores, mas, se não for capaz de neutralizá-los por completo, pode continuar indefinidamente (29). 
A seguir, as células musculares lisas começam a migrar da camada média para a íntima, se proliferam e secretam colágeno, dando origem à lesão intermediária. Nesta fase, o espessamento da íntima provoca o remodelamento, ou seja, uma dilatação da artéria compensatória ao estreitamento do lúmen. Mais uma vez, sob o estímulo da LDLox, entre outros, as células do sistema imune local liberam enzimas, citocinas e fatores de crescimento que podem induzir necrose focal. Ciclos repetidos de acúmulo e ativação de células mononucleares, migração e proliferação das células musculares lisas com produção de colágeno, levam ao aumento progressivo da lesão, até que se estruture uma capa fibrosa ao redor de um núcleo lipídico e de tecido necrótico, a chamada lesão avançada. Quando a artéria já não consegue se dilatar adequadamente, a lesão pode então alterar o fluxo sanguíneo, manifestando-se clinicamente por síndromes coronarianas estáveis (29).

O excesso de células espumosas no core lipídico torna a placa mais frágil, sendo que uma fratura na capa fibrosa, com conseqüente formação de trombo, pode complicar a lesão avançada, sendo considerada a principal causa de eventos cardiovasculares agudos. Esta capa fibrosa sofre remodelamento dinâmico e contínuo, sendo que o balanço entre os processos de síntese e degradação dos seus componentes é controlado por mediadores inflamatórios, liberados em parte como resposta à LDLox. As citocinas podem induzir a expressão de metaloproteinases da matriz extracelular, enzimas que digerem as fibras de colágeno e elastina, fragilizando a capa da lesão (50). Macrófagos em cultura, tratados com LDLox, exibiram aumento na expressão da metaloproteinase tipo 9 , assim como redução da expressão do inibidor tissular desta enzima (5l).

Outro ponto de interesse é a população de células musculares lisas da placa. Sítios de trombose fatal onde as placas se rompem por falha mecânica, tipicamente têm poucas células musculares lisas. A morte destas células, responsáveis pela manutenção e reparo da matriz extracelular, pode ser desencadeada por estímulos inflamatórios locais (50). Comentamos previamente que a captação de LDLox por receptores scavenger das células musculares lisas induz a apoptose celular (33), implicada na desestabilização e tendência à ruptura da lesão aterosclerótica. Cabe ressaltar que os eventos aterotrombóticos são mais comuns em bifurcações arteriais, onde o turbilhonamento do fluxo sanguíneo confere maior estresse mecânico, facilitando o rompimento das placas instáveis.

Uma minoria dos eventos agudos decorre de erosão da superfície do endotélio. Neste caso, são as células endoteliais que sofrem apoptose sob estímulo inflamatório, ou secretam proteinases estimuladas pela LDLox, favorecendo a lesão da íntima. Nestas circunstâncias, o desequilíbrio entre as propriedades prótrombóticas e fibrinolíticas do endotélio favorece a trombogênese. As células endoteliais expressam fator tissular pró-coagulante e aumentam a produção do inibidor do ativador do plasminogênio (PAI-1). O processo inflamatório presente no endotélio da lesão aterosclerótica diminui sua produção de óxido nítrico (NO); adicionalmente, as ROS podem neutralizar seu efeito vasodilatador provocando vasoespasmo, ou diminuir sua ação antiinflamatória na via do $\mathrm{NF \kappa B}$, ou, ainda, inibir seus efeitos anti-agregação plaquetária. Conjuntamente, estas ações concorrem para o desenvolvimento do trombo no ateroma sob estresse oxidativo (50). A figura 1 esquematiza a instabilização e trombose da placa aterosclerótica.

A presença da LDLox associa-se à vulnerabilidade das placas ateroscleróticas. Estudo envolvendo pacientes coronariopatas encontrou níveis séricos de LDLox mais elevados nos portadores de infarto agudo do miocárdio do que naqueles com angina instável ou estável, correlacionando-a com a gravidade do quadro (52). Na mesma linha, altas concentrações de LDLox no plasma ou na placa de pacientes submetidos à endarterectomia carotídea associaram-se à tendência à ruptura das lesões (53). Outros autores encontraram, ainda, aumento nos níveis de LDLox e de seus marcadores - o anti-LDLox e imunocomplexos antiLDL/LDLox - na síndrome coronariana aguda, refletindo uma possível liberação da LDLox na circulação e uma resposta imune exacerbada (54). Dessa forma, é provável que estas determinações possam se

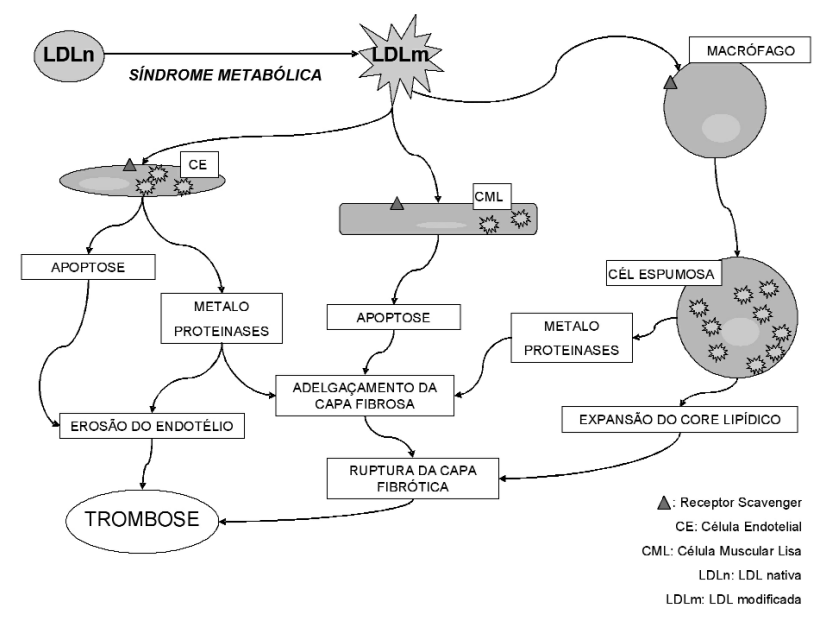

Figura 1. participação da LDL modificada na instabilização e trombose da placa ateromatosa 
tornar úteis no diagnóstico de eventos cardiovasculares agudos. No contexto da SM, a LDLox foi, ainda, pouca explorada e mais estudos são desejáveis para fixar seu papel como marcador de risco cardiovascular.

\section{Considerações finais}

Apesar dos avanços no estudo do perfil lipídico, a LDL continua sendo um dos principais fatores de risco cardiovascular e o mais amplamente utilizado. No entanto, uma das limitações do emprego desta lipoproteína refere-se ao fato de que o aumento da sua concentração sanguínea não é suficiente para explicar a ocorrência de síndromes agudas. Isso denota que as alterações qualitativas da partícula, ou seja, sua composição química e seu poder de agressão à parede arterial, têm importância fundamental para o entendimento de tais eventos. A presente revisão resgatou o conhecimento sobre a participação da LDL, desde as primeiras modificações ocorridas na vigência da SM, até seu papel na formação e instabilização da placa aterosclerótica e trombose aguda.

Muitos dados provêm de estudos experimentais, principalmente no que se refere à $\operatorname{LDL}(-)$ ou minimamente modificada. Várias metodologias vêm sendo desenvolvidas para a dosagem da LDLox in vivo, dos seus anticorpos ou imunocomplexos, mas ainda são raros os relatos de sua importância como preditora de risco cardiovascular na SM. É imprescindível a realização de estudos epidemiológicos prospectivos para comprovar esta relação.

A LDLpd, ou padrão $B$, foi mais comumente estudada em coortes, sendo incontestável sua presença na dislipidemia da SM e sua relação com risco cardiovascular. Porém, na prática clínica, sua determinação ainda é pouco empregada por limitações técnicas.

Em resumo, a presença marcante da LDLm em todas as etapas da aterogênese reforça a importância de se delinear estudos mais amplos para esclarecer seu papel, particularmente no contexto da SM. Também, alerta-se para a necessidade de uniformização dos termos até agora empregados e do desenvolvimento de métodos simples para a determinação de LDLs modificadas, a fim de que possam ser mais amplamente utilizadas na prática clínica.

\section{REFERÊNCIAS}

1. Executive Summary of the Third Report of The National Cholesterol Education Program (NCEP) expert panel on detection, evaluation, and treatment of high blood cholesterol in adults (Adult Treatment Panel III). JAMA 2001;285:2486-97.
2. Puavilai $G$, Chanprasertyotin S, Sriphrapradaeng A. Diagnostic criteria for diabetes mellitus and other categories of glucose intolerance: 1997 criteria by the Expert Committee on the Diagnosis and Classification of Diabetes Mellitus (ADA), 1998 WHO consultation criteria, and 1985 WHO criteria. World Health Organization. Diab Res Clin Pract 1999;44:21-6.

3. Einhorn D, Reaven GM, Cobin RH, Ford E, Ganda OP, Handelsman $Y$, et al. American College of Endocrinology position statement on the insulin resistance syndrome. Endocr Pract 2003:9:237-52.

4. Alberti KG, Zimmet P, Shaw J. The metabolic syndrome - a new worldwide definition. Lancet 2005;366:1059-62.

5. St-Pierre AC, Ruel IL, Cantin B, Dagenais GR, Bernard MP, Despres JP, et al. Comparison of various electrophoretic characteristics of LDL particles and their relationship to the risk of ischemic heart disease. Circulation 2001;104:2295-9.

6. Wajchenberg BL. Subcutaneous and visceral adipose tissue: their relation to the metabolic syndrome. Endocr Rev 2000;21:697-738.

7. Brunzell JD, Hokanson JE. Dyslipidemia of central obesity and insulin resistance. Diabetes Care 1999;22(suppl 3): C10-3.

8. Carr MC, Brunzell JD. Abdominal obesity and dyslipidemia in the metabolic syndrome: importance of type 2 diabetes and familial combined hyperlipidemia in coronary artery disease risk. J Clin Endocrinol Metab 2004;89:2601-7.

9. Lamarche B, Tchernof A, Mauriege P, Cantin B, Dagenais GR, Lupien PJ, et al. Fasting insulin and apolipoprotein B levels and low-density lipoprotein particle size as risk factors for ischemic heart disease. JAMA 1998;279:1955-61.

10. Lamarche B, Tchernof A, Moorjani S, Cantin B, Dagenais GR, Lupien PJ, et al. Small, dense low-density lipoprotein particles as a predictor of the risk of ischemic heart disease in men. Prospective results from the Quebec Cardiovascular Study. Circulation 1997:95:69-75.

11. Zambon A, Hokanson JE, Brown BG, Brunzell JD. Evidence for a new pathophysiological mechanism for coronary artery disease regression: hepatic lipasemediated changes in LDL density. Circulation 1999; 99:1959-64.

12. Stryer L. Biosynthesis of membrane lipids and steroids. In: Stryer L, editor. Biochemistry. $4^{\text {th }}$ edition. New York: WH Freeman, 1996, p.697-8.

13. Mahley RW, Weisgraber KH, Farese RV. Disorders of lipid metabolism. In: Williams textbook of endocrinology, $10^{\text {th }}$ edition. New York: Elservier Science, 2003, p.1646-52.

14. Chang YH, Abdalla DS, Sevanian A. Characterization of cholesterol oxidation products formed by oxidative modification of low-density lipoprotein. Free Radic Biol Med 1997:23:202-14.

15. Yla-Herttuala S, Palinski W, Rosenfeld ME, Parthasarathy $\mathrm{S}$, Carew TE, Butler $\mathrm{S}$, et al. Evidence for the presence of oxidatively modified low-density lipoprotein in atherosclerotic lesions of rabbit and man. J Clin Invest 1989;84:1086-95. 
16. Henriksen T, Mahoney EM, Steinberg D. Enhanced macrophage degradation of low density lipoprotein previously incubated with cultured endothelial cells: recognition by receptors for acetylated low density lipoproteins. Proc Natl Acad Sci USA 1981;78:6499-503.

17. Henriksen T, Mahoney EM, Steinberg D. Enhanced macrophage degradation of biologically modified low density lipoprotein. Arteriosclerosis 1983;3:149-59.

18. Parthasarathy S, Printz DJ, Boyd D, Joy L, Steinberg D. Macrophage oxidation of low-density lipoprotein generates a modified form recognized by the scavenger receptor. Arteriosclerosis 1986;6:505-10.

19. Steinberg D, Parthasarathy S, Carew TE, Khoo JC, Witztum JL. Beyond cholesterol. Modifications of lowdensity lipoprotein that increase its atherogenicity. $\mathbf{N}$ Engl J Med 1989;320:915-24.

20.Kovanen PT, Pentikainen MO. Circulating lipoproteins as proinflammatory and anti-inflammatory particles in atherogenesis. Curr Opin Lipidol 2003;14:41 1-9.

21. Scheffer PG, Teerlink T, Heine RJ. Clinical significance of the physicochemical properties of LDL in type 2 diabetes. Diabetologia 2005;48:808-16.

22. Avogaro P, Bon GB, Cazzolato G. Presence of a modified low density lipoprotein in humans. Arteriosclerosis 1988;8:79-87.

23. Sanchez-Quesada JL, Benitez S, Ordonez-Llanos J. Electronegative low-density lipoprotein. Curr Opin Lipidol 2004; 15:329-35.

24. Berliner JA, Territo MC, Sevanian A, Ramin S, Kim JA Bamshad B, et al. Minimally modified low density lipoprotein stimulates monocyte endothelial interactions. J Clin Invest 1990;85:1260-6.

25. Austin MA, Breslow JL, Hennekens $\mathrm{CH}$, Buring JE, Willett WC, Krauss RM. Low-density lipoprotein subclass patterns and risk of myocardial infarction. JAMA 1988;260:1917-21.

26. Sevanian A, Hwang J, Hodis $H$, Cazzolato G, Avogaro $P$, Bittolo-Bon $G$. Contribution of an in vivo oxidized $L D L$ to LDL oxidation and its association with dense LDL subpopulations. Arterioscler Thromb Vasc Biol 1996; 16:784-93.

27. Sanchez-Quesada JL, Perez A, Caixas A, OrdonezLlanos J, Carreras $G$, Payes A, et al. Electronegative low density lipoprotein subform is increased in patients with short-duration IDDM and is closely related to glycaemic control. Diabetologia 1996:39:1469-76.

28. Sanchez-Quesada JL, Perez A, Caixas A, Riglas M, Payes A, Benitez $S$, et al. Effect of glycemic optimization on electronegative low-density lipoprotein in diabetes: relation to nonenzymatic glycosylation and oxidative modification. J Clin Endocrinol Metab 2001;86:3243-9.

29. Ross R. Atherosclerosis - an inflammatory disease. $\mathbf{N}$ Engl J Med 1999;340:115-26.

30. Lopes-Virella MF, Virella G. Immune mechanisms of atherosclerosis in diabetes mellitus. Diabetes 1992; 41 (suppl 2):86-91.

31. Chen M, Masaki T, Sawamura T. LOX-1, the receptor for oxidized low-density lipoprotein identified from endothelial cells: implications in endothelial dysfunction and atherosclerosis. Pharmacol Ther 2002;95:89-100.
32. Cominacini L, Pasini AF, Garbin U, Davoli A, Tosetti ML, Campagnola $\mathrm{M}$, et al. Oxidized low-density lipoprotein (Ox-LDL) binding to ox-LDL receptor-1 in endothelial cells induces the activation of NF-kappaB through an increased production of intracellular reactive oxygen species. J Biol Chem 2000;275: 12633-8.

33. Kataoka $\mathrm{H}$, Kume N, Miyamoto S, Minami M, Morimoto $\mathrm{M}$, Hayashida K, et al. Oxidized LDL modulates Bax/Bcl-2 through the lectinlike Ox-LDL receptor-1 in vascular smooth muscle cells. Arterioscler Thromb Vasc Biol 2001:21:955-60.

34. Horkko S, Binder CJ, Shaw PX, Chang MK, Silverman G, Palinski W, et al. Immunological responses to oxidized LDL. Free Radic Biol Med 2000:28:1771-9.

35. Reaven PD, Napoli C, Merat S, Witztum JL. Lipoprotein modification and atherosclerosis in aging. Exp Gerontol 1999:34:527-37.

36. Palinski W, Ord VA, Plump AS, Breslow JL, Steinberg D, Witztum JL. ApoE-deficient mice are a model of lipoprotein oxidation in atherogenesis. Demonstration of oxidation-specific epitopes in lesions and high titers of autoantibodies to malondialdehyde-lysine in serum. Arterioscler Thromb 1994:14:605-16.

37. Orchard TJ, Virella G, Forrest KY, Evans RW, Becker DJ, Lopes-Virella MF. Antibodies to oxidized LDL predict coronary artery disease in type 1 diabetes: a nested case-control study from the Pittsburgh Epidemiology of Diabetes Complications Study. Diabetes 1999;48:1454-8.

38. Shoji T, Nishizawa Y, Fukumoto M, Shimamura K, Kimura $\mathrm{J}$, Kanda $\mathrm{H}$, et al. Inverse relationship between circulating oxidized low density lipoprotein (OxLDL) and anti-OxLDL antibody levels in healthy subjects. Atherosclerosis 2000; 148:171-7.

39. Salonen JT, Yla-Herttuala S, Yamamoto R, Butler S, Korpela $\mathrm{H}$, Salonen $\mathrm{R}$, et al. Autoantibody against oxidised LDL and progression of carotid atherosclerosis. Lancet 1992;339:883-7.

40. Tornvall P, Waeg G, Nilsson J, Hamsten A, Regnstrom J. Autoantibodies against modified low-density lipoproteins in coronary artery disease. Atherosclerosis 2003: 167:347-53

41. Faviou E, Vourli G, Nounopoulos C, Zachari A, Dionyssiou-Asteriou A. Circulating oxidized low density lipoprotein, autoantibodies against them and homocysteine serum levels in diagnosis and estimation of severity of coronary artery disease. Free Radic Res 2005:39:419-29.

42. De Castellarnau C, Sanchez-Quesada JL, Benitez S, Rosa R, Caveda L, Vila L, et al. Electronegative LDL from normolipemic subjects induces IL-8 and monocyte chemotactic protein secretion by human endothelial cells. Arterioscler Thromb Vasc Biol 2000:20:2281-7.

43. Sanchez-Quesada JL, Camacho M, Anton R, Benitez S, Vila L, Ordonez-Llanos J. Electronegative LDL of FH subjects: chemical characterization and induction of chemokine release from human endothelial cells. Atherosclerosis 2003; 166:261-70.

44. Benitez S, Sanchez-Quesada JL, Ribas V, Jorba $O$, Blanco-Vaca F, Gonzalez-Sastre F, et al. Plateletactivating factor acetylhydrolase is mainly associated with electronegative low-density lipoprotein subfraction. Circulation 2003; 108:92-6. 
45. Brand K, Page S, Rogler G, Bartsch A, Brandl R, Knuechel $R$, Page $M$, et al. Activated transcription factor nuclear factor-kappa B is present in the atherosclerotic lesion. $\mathbf{J}$ Clin Invest 1996:97:1715-22.

46. Dandona P, Aljada A, Mohanty P, Ghanim H, Hamouda $W$, Assian $E$, et al. Insulin inhibits intranuclear nuclear factor kappaB and stimulates IkappaB in mononuclear cells in obese subjects: evidence for an antiinflammatory effect? J Clin Endocrinol Metab 2001; 86:3257-65.

47. Hodis HN, Kramsch DM, Avogaro P, Bittolo-Bon G, Cazzolato $G$. Hwang J, et al. Biochemical and cytotoxic characteristics of an in vivo circulating oxidized lowdensity lipoprotein (LDL-). J Lipid Res 1994;35:669-77.

48. Demuth K, Myara I, Chappey B, Vedie B, PechAmsellem MA, Haberland ME, et al. A cytotoxic electronegative LDL subfraction is present in human plasma. Arterioscler Thromb Vasc Biol 1996; 16:773-83.

49. Chen $\mathrm{CH}$, Jiang T, Yang JH, Jiang W, Lu J, Marathe GK, et al. Low-density lipoprotein in hypercholesterolemic human plasma induces vascular endothelial cell apoptosis by inhibiting fibroblast growth factor 2 transcription. Circulation 2003; 107:2102-8.

50. Libby P. Current concepts of the pathogenesis of the acute coronary syndromes. Circulation 2001;104:365-72.

51. Xu XP, Meisel SR, Ong JM, Kaul S, Cercek B, Rajavashisth TB, et al. Oxidized low-density lipoprotein regulates matrix metalloproteinase-9 and its tissue inhibitor in human monocyte-derived macrophages. Circulation 1999:99:993-8.
52. Ehara S, Ueda M, Naruko T, Haze K, Itoh A, Otsuka M, et al. Elevated levels of oxidized low-density lipoprotein show a positive relationship with the severity of acute coronary syndromes. Circulation 2001; 103:1955-60.

53. Nishi $K$, Itabe $H$, Uno $M$, Kitazato KT, Horiguchi $H$, Shinno $\mathrm{K}$, et al. Oxidized LDL in carotid plaques and plasma associates with plaque instability. Arterioscler Thromb Vasc Biol 2002;22: 1649-54.

54. Tsimikas S, Bergmark C, Beyer RW, Patel R, Pattison J, Miller $E$, et al. Temporal increases in plasma markers of oxidized low-density lipoprotein strongly reflect the presence of acute coronary syndromes. J Am Coll Cardiol 2003:41:360-70.

\section{Endereço para correspondência}

Sandra Roberta $G$. Ferreira

Departamento de Medicina Preventiva

Universidade Federal de São Paulo

Rua Botucatu 740

04023-062 São Paulo, SP

Fax: (11) 5571-5000

E-mail: ferreira@medprev.epm.br 